\title{
ACTIVE CONTROLS AND NON-INVASIVE MONITORING FOR HIGH SPEED TRAINS
}

\author{
Aldo Balestrino, Ottorino Bruno, Alberto Landi, Luca Sani \\ Department of Electrical Systems and Automation, University \\ of Pisa, via Diotisalvi 2, 56126, Pisa, Italy \\ phone: +390502217300 - fax: +390502217333 - e-mail: landi@ieee.org
}

\begin{abstract}
International interest is growing for improving performance of the rail transport system. A crucial problem for railway companies is to prevent damages to the overhead line equipment and to pantographs. A brief overview of our research activities is addressed, in the fields of active pantographs and advanced methods for measuring the quality of the current collection for high-speed trains. The pantograph-overhead contact wire interaction is investigated by using a phototube sensor, wavelet transform applied to the line current and infrared camera. The results obtained are relevant for monitoring the quality of the current collection and for a predictive maintenance of the pantograph and of the contact line. Copyright (C) 2005 IFAC
\end{abstract}

Keywords: Railways, Maintenance, Monitoring, Active pantographs, Wavelets, Infrared detectors.

\section{INTRODUCTION}

The development of innovative methods for improving efficiency, reliability and safety in railways is a primary objective of the railway research. Highspeed trains need a regular current transmission in pantograph-catenary systems. European Standards about interoperability (96/48/EC) define the rules for an efficient circulation of high-speed trains in Europe, i.e., a locomotive must be designed to freely operate inside European countries. A high quality current collection is characterised by a continuous smooth contact between the pantograph and the overhead contact wire. A poor contact produces various drawbacks, including bursts of arcing: if they have a long duration, locomotive efficiency may reduce and an excessive wear of the pantograph strips and of the contact wire may lead to maintenance problems, up to crashing of catenary. A primary objective in railway research is the reduction of contact forces: a first interesting solution is the design of innovative pantographs with reduced head mass and active control. All railway companies are well aware of the problems affecting the current collection: a relevant objective both for academic researchers and for railway companies is the development of innovative pantographs and the application of new sensors for a continuous monitoring of the sliding contact and for helping maintenance activities. In the past, research activity in railways have been restricted to expert engineers and it is spread through specific journals, usually published by the respective national railway companies, e.g., Ingegneria Ferroviaria (sponsored by Italian Railways), Elektrische Bahnen (sponsored by German Railways), Revue Générale des Chemin de Fer (sponsored by French Railways), Quarterly Report of the Railway Technical Research Institute (sponsored by Japanese Railways). International independent journals are few: among them important are the Proceedings of the Institution of Mechanical Engineers, Part F - Journal of Rail and Rapid Transit and Vehicle System Dynamics, the last one only partially devoted to the railway research. In fact the greater part of the scientific research is developed inside own institutions of the railway companies (e.g.,UIC, SNCF Direction de l'Ingegnerie a Parigi, The Railway Technical Research Institute in Tokio, Korea Railroad Research Institute, ARRIAustralian Railway Research Institute, The European Rail Research Institute in Holland, the Advanced 
Rail Research Centre in Sheffield, the ITALFERR in Roma). The augmented interests in railway researches, due to the interoperability standards is demonstrated from the birth of many new international conferences, e.g., the series Computers in Railways, or the World Congresses on Railway Research (WCRR).

Therefore in recent years many universities have devoted their activities to deal problems relative to the rail transportation, both in Italy and in different countries, as Germany, France, Great Britain and Japan, considered leaders in development of railway technologies. In Italy the Ministry of Research financed in 2000 the biennial project of research: "Innovative Controls in High Speed Transport Systems" and the operating unit of Pisa held the national coordination: the results of the project have been internationally appreciated, especially for the contribution in monitoring pantograph-catenary interaction. The main result of Pisa group was the study and implementation of phototube UV sensors, mounted on the deck top of a measurement coach, effective in detecting break arcs on line. A correlation of measured repetitive break arcs with the kilometric progression of the railway line Rome-Florence revealed its validity for an automatic checking of the status of the contact overhead line. These results, obtained by a strict cooperation with the Unit of Technologies and Rolling Stocks of Florence - Italian Railways, proved to be a useful tool for helping maintenance activities both for the overhead line and for the pantograph. The results obtained are meaningful, if compared with the international scenario of innovative research for maintenance: Japanese Railways have developed the Dr Yellow, a diagnostic train for maintenance on the Tokyo-Osaka line, whose sensors of arcing are based on UV phototube sensors, just similar to the sensors proposed in our research activity. In the next sections a brief overview of the main results obtained from the group of Pisa is addressed, along with some remarks on the perspectives.

\section{ACTIVE PANTOGRAPHS.}

For high-speed electric railway traction, the current is collected by one or two pantographs in a sliding contact with the overhead wire, which is supported by means of vertical droppers suspended from a catenary wire (excluding MAGLEV systems and British Railways, where usually a third rail is used as a feeder). In the ideal hypothesis of overhead wires stiff, rectilinear and parallel to the railway line, the moving pantograph exerts a constant contact force providing a perfect current collection. Such hypothesis is an illusion: stiffness of the overhead wire varies from a maximum near the support towers to a minimum in the middle of the span and a similar (quantitatively scaled) phenomenon occurs between any couple of adjacent droppers. Therefore near the support towers (and near the droppers) the pantograph is forced downward quickly, which results in a reduction of contact forces or even a loss in contact with the overhead wire: an electrical arcing may occur, causing the overhead wire and the pantograph shoe to deteriorate rapidly. Furthermore, irregular laying, different thermal dilatation, atmospheric conditions (e.g. wind, rain), and even perturbations due to the movement itself are examples of a real scenario far from the ideal case. The effects of the interaction between the overhead line and the moving pantograph are more severe at high speed (over $250 \mathrm{~km} / \mathrm{h}$ ); in order to reduce losses of contact or excessive oscillations of the catenary, the standard current pickup equipment must be modified. In the past, a number of simple and ingenious expedients has been adopted to improve the performance of the pantograph-catenary system. The simplest one (today still widely used in traditional trains to avoid loss of contact) is to increase the uplift contact force between the pantograph and the catenary. Unfortunately for high-speed trains the lifetime of the collector strips and particularly of the contact wire reduces dramatically, because of the deterioration due to erosion and abrasion. On the other hand more complex and expensive solutions have been suggested, leading to a new design of the overhead system. Many authors proposed as an alternative solution a reduction of the pantograph head mass in order to minimise the losses of contact. Over the years some studies have been devoted to a different approach: instead of modifying the geometrical and/or the mechanical parameters of the overhead wire structure and of the pantograph, active pantographs may be introduced. Now there seems to be a general agreement that the design of high performance pantographs offers the best potentiality for the future in terms of costs and optimal current transmission (Poetsch et al., 1997). In spite of the great variety of proposed solutions, a major problem to overcome is the practical application of the theoretical (or simulated) results. Every solution must satisfy the requirements of safety, reliability, simplicity and must be technically as well as economically feasible.

\subsection{Active control strategies.}

Various control strategies were considered: a first conclusion was that the control action has to maintain the uplift contact force between the overhead wire and the pantograph head almost constant. From several simulation tests it was verified that even the simple solution represented by a standard proportional controller was effective for guaranteeing a correct current collection in the case of high-speed trains. In practice, the aim of the control action is to maintain the average uplift contact force at a value over $70 \mathrm{~N}$ in order to guarantee a correct current transmission and to avoid excessive wear.

Practical drawbacks are:

- current transmission capability has to be guaranteed even if a failure occurs in the active control apparatus,

- the uplift contact force has to be determined possibly avoiding a force sensor.

The pantograph can be controlled in two different ways (see Fig. 1): as a first solution the control force 
$\mathrm{F}_{\mathrm{q}}$ acts on the lower arms of the pantograph; in the second one the control torques $C_{1}$ and $C_{2}$ are applied directly to the upper arms.

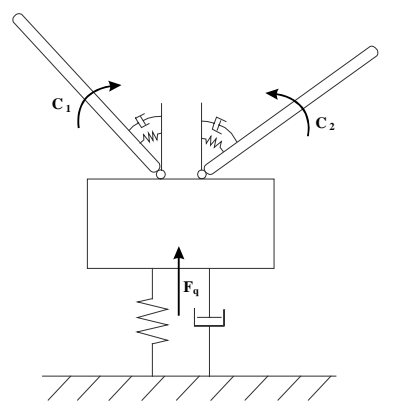

Fig. 1. Control actions for an active pantograph.

In order to overcome the first drawback, the uplift contact force can be subdivided into two terms: a mean term given by a constant force, provided by the traditional passive mechanism (usually a spring or a pneumatic cylinder) as in standard pantographs and an additional variable term, provided by a closedloop active actuator. In such a way the current collection capability is maintained even in the case of a failure in the active control apparatus. Extensive simulations have been performed, for comparing the two different control strategies: both active strategies can limit the probability of losing contact. The best solution must fulfil the requirements of safety, reliability and simplicity in a practical application.

A practical implementation of the active pantograph is suggested in Balestrino et al., (2000), denoted as "wire actuated control".

In figure 2 a schematic drawing of the wire-actuated control is shown in case of an asymmetric pantograph. The control action is exerted on each upper arm through a wire. The wire can wind or unwind due to the action of an electric motor located on the roof of the vehicle. The wire acts only in traction, i.e. the contact force can be regulated if and only if the wire is under tension. In order to satisfy such condition the contact force provided by the passive mechanism is set to a higher value than in the standard case: the control action of the wire will regulate the contact force to the desired level, pulling down the upper arms. The speed of response of the control system is very high: only the upper arms are controlled, reducing the mass to be moved with respect to the case of the control force acting on the linkage frame and moving the mass of the whole pantograph. A brushless DC motor with low inertia and small electro-mechanical time constant could be the simplest choice for the electrical actuator.

An advantage of the proposed solution is the reduced structural modification with respect to conventional pantographs; it is independent from the pantograph geometry, so that it could be applied to different existing pantograph topologies. Unfortunately, in order to close the control loop a measurement of the contact force applied to the pantograph head is required. Such requirement could be an obstacle for a practical implementation of the closed loop control: a technical solution based on the observed contact force is proposed, but solutions based on the application of a Kalman filter (Diana et al., 1998) can be successfully adopted.

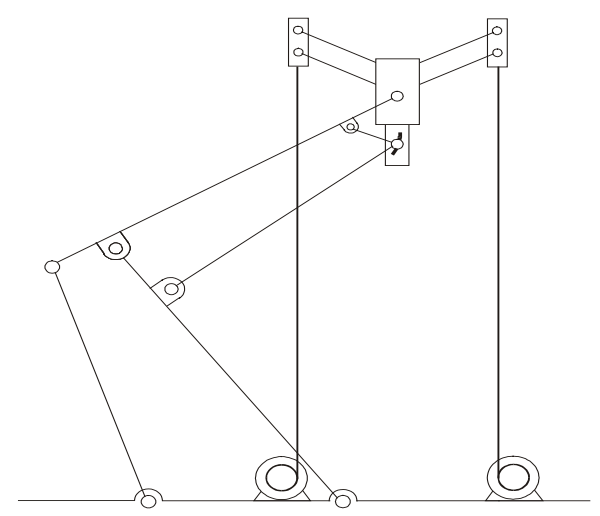

Fig. 2. Wire actuated control scheme.

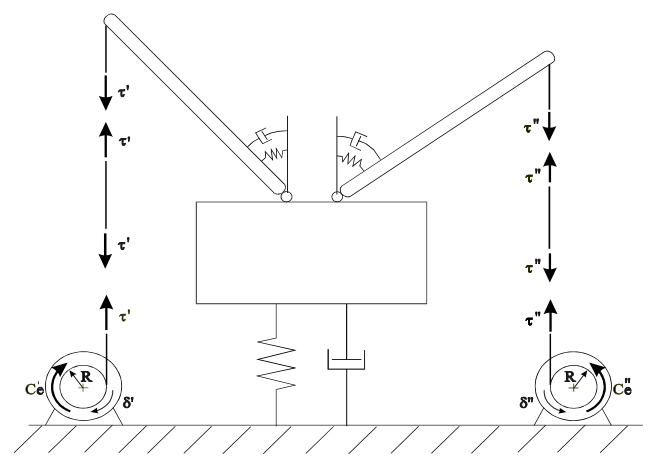

Fig. 3. Wire actuated control scheme: lumped model

\section{SENSORS FOR A CONTINUOUS MONI- TORING OF THE SLIDING CONTACT}

High quality current collection is characterized by a continuous contact between the pantograph and the overhead line. Poor contact behaviour produces various drawbacks, including arcing, excessive wear of the pantograph strips and of the contact wire, bouncing against swivel registration arms and fixed parts of the overhead line. High speeds worsen these negative aspects: wires start oscillating and the pantograph head cannot stay in contact, particularly in case of height variations, e.g., entering a tunnel. A pair of pantographs on a train worsens the problem of oscillating overhead line for the rear pantograph: it encounters an overhead line already excited by the first one, so that the quality of the contact deteriorates. All railway companies have to set up and test monitoring systems for checking the status of the line and planning maintenance activities.

A traditional method consists of periodic inspections along the line, by using inspection vehicles equipped for catenary checking. This method has two main drawbacks. First it is expensive, because it requires that the line be maintained out of service during inspections, usually performed at night, when the frequency of the trains running along the line is reduced. Second, it allows only a limited activity for a few kilometres during every inspection. Therefore many companies are developing new specialised 
equipment for high-speed operations. A different philosophy is devoted to perform on-line tests: checking activity may be performed by adding a measurement section to standard passenger (or freight) trains for a continuous monitoring of the line. In the sequel three different solutions are considered, for a non-invasive on-line monitoring of the contact between the overhead electrified contact line and the pantograph.

\subsection{Phototube sensors.}

In Bruno et al. (2002) the performance of a new measurement system was described in detail. It is based on phototube sensors, for detecting arcing between the pantograph and the contact line. Test runs were performed on board of high-speed trains of Italian Railways, with a double pantograph and an acquisition system on board of a measurement coach. Twin phototube sensors were physically placed on the top deck of the locomotive, in front and rear the pantograph, for detecting the presence of UV emissions, with an output signal binary, producing square wave signals. A relevant outcome of the research was the kilometre-by-kilometre detection of arcing. A statistical analysis was performed, related both to entering and to exiting periods of arcing separately, to put into evidence a distribution of the number of arcing occurrences per kilometre.

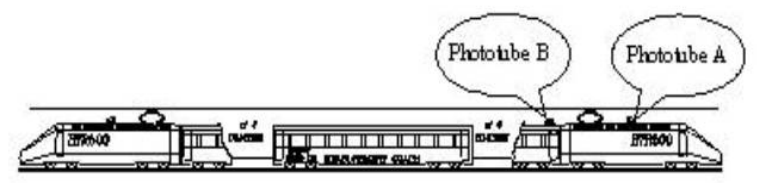

Fig. 4. Phototubes location for arcing detection

This analysis was able to discriminate an index for defining whether the overhead line was affected by arcing or by the so-called electric welding effect. This effect is due to a defective sliding contact, because of the roughness of the contact wire and of the collector strips, mainly due to wear. Therefore, hot spots and micro-welding phenomena occur along the contact surfaces. If the train is running at high-speed (the higher the speed, the more critical the effects) the welded spots are instantaneously broken off and the contact wire further deteriorates. In such cases the wear of the collector strips increases and continuous arcing occurs. Therefore, the welding effect produces a sequence of continuous sparking, damaging the overhead contact line and increasing the rapid maintenance burden. It was verified that the phototube was the only sensor, along with the human eye, able to detect such an effect. It was checked that an analysis of data collected from the twin phototube sensor reveals critical points of the overhead line and constitutes a reliable information for predicting an excessive wire wear and for helping quick maintenance activities. Advantages of the system proposed can be summarized as:

1. quick detection of singular points of the overhead line from the presence of repeated break arcs for different test runs;
2. quick detection of long deteriorations of the overhead line in the presence of the electric welding effect;

3. definition of an index for evaluating the quality of the current collection;

4. preventive actions can be planned for avoiding damages to the line and for improving the regularity and the safety of the railway traffic, especially in high speed running lines.

\subsection{Wavelet analysis.}

A drawback of phototube sensors is that dedicated sensors are needed: phototubes for arcing detection are prototypes not commercially available. It would be better to consider an analysis of data more commonly acquired, for example line current measurements. The problem is that current measurements often hide useful information: harmonic currents could recover correlations with periods of arcing, but the standard Fourier analysis has the drawback of losing time information in case of transient signals, like arcing. A short time Fourier analysis, i.e., a harmonic decomposition restricted to a limited sliding window of data could partially avoid such problem, but wavelet analysis represents a more suitable method for checking transient unpredictable phenomena, like the occurrence of arcing. Multiresolution wavelet analysis to the signals acquired from the measurement coach was applied, in order to investigate whether the wavelet transform analysis can extract useful information for monitoring the status of the overhead line, detecting the losses of contact between the pantograph and the overhead line. The total tractive current was analysed, using data acquired from an Italian high-speed train. The train was equipped with an equipotential wire (EW), i.e., a conductor connecting the front and the rear pantographs, able to compensate for the loss of contact by means of transferring current supply, whenever one of the two pantographs loses contact. It can be noted that such a device represents a selective and precise sensor able to detect the losses of contact (i.e., a sudden loss of contact relative to one pantograph always produces a fast variation in the current conducted by the EW). Due to the presence of the EW during the high-speed test runs, the results obtained from the phototube sensors and from a wavelet analysis have been validated, correlating them with the data collected from the EW itself. Detailed results of such an application were described in Barmada et al. (2003).

As an example of the results obtained, consider data acquisitions shown in fig.5: each plot is referred to one kilometre test run, at an average speed of 220 $\mathrm{km} / \mathrm{h}$. The upper two subplots show the duration (in $\mathrm{ms}$ ) of arcing periods detected from the front and from the rear phototube, respectively. In the lower subplots the total line current absorbed and the current from the equipotential wire $(\mathrm{EW})$ are shown, both in $\mathrm{kA}$. 


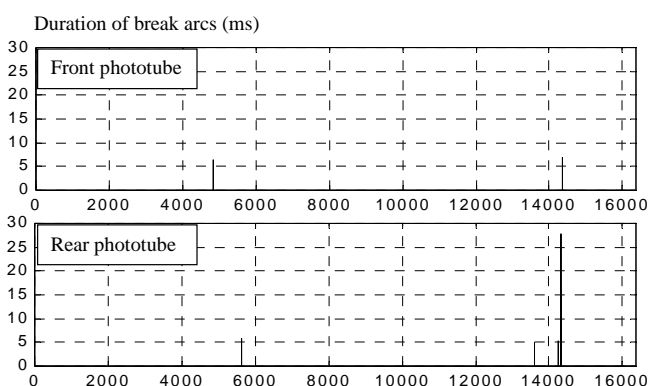

Total line current $(\mathrm{kA})$
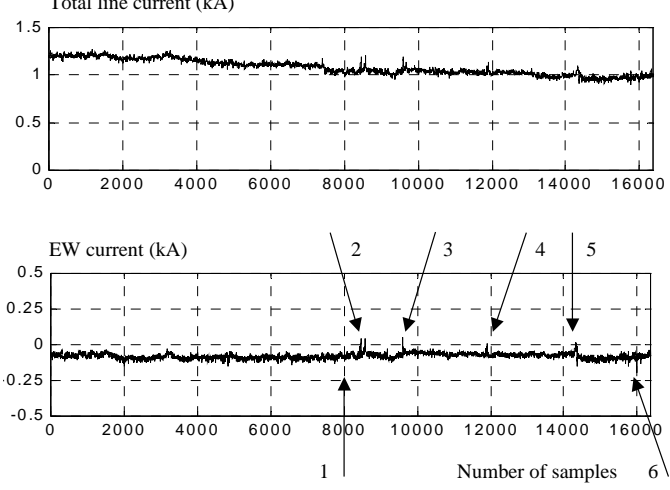

Fig. 5. Signal acquisitions: case of a single relevant break arc.

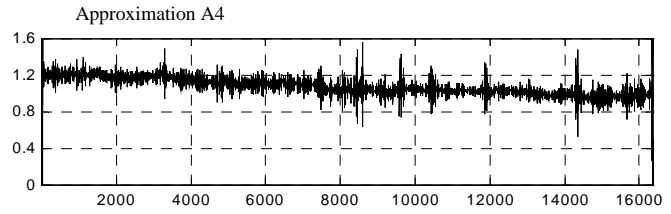

Detail D4

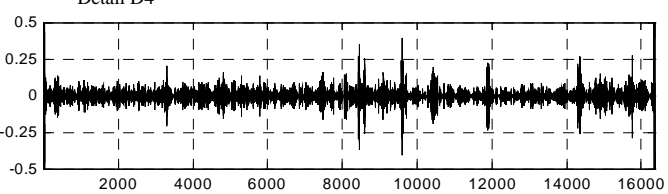

Detail D3

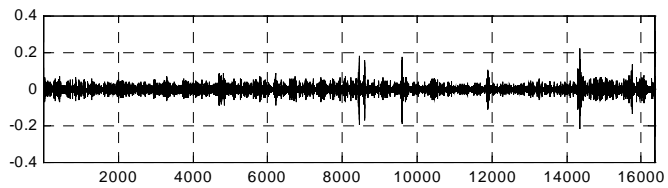

Detail D2

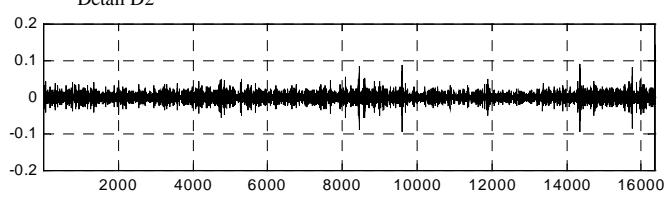

Detail D1

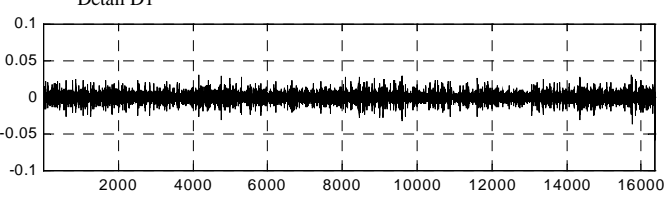

Fig. 6. Wavelet decomposition of the traction current: case of a single relevant break arc.

Note that arcing is detected only from the equipotential wire data acquisition in cases of losses of contact of the unmonitored second pantograph. As a convention, losses of contact on the unmonitored pantograph correspond to positive current peaks measured from the EW; negative peaks correspond to losses of contact on the monitored pantograph. Each plot is a function of the number of samples. Arrows mark losses of contact revealed from the EW.

The wavelet decomposition of the traction current is shown in fig. 6.

A relevant phenomenon (single break arc) is revealed in all details, representing arcing revealed both by the phototube and via the EW (arrow 5). Four periods of arcing (corresponding to arrows 1, 2, 3, 4 of the EW acquisition) on the unmonitored pantograph are revealed by the wavelet analysis: none of them has a fast transient, because of their negligible effects on the lower details. The last arrow 6 is revealed both from the EW acquisition and from the wavelet analysis, but it is undetected from the phototube sensor. It may be considered as an example of arc of small duration, undetected from the phototube sensor. Note that all relevant phenomena are pointed with arrows in the plot representing EW currents, but the wavelet analysis is performed on the total line current where such phenomena are unclear to detect at a first glance.

\subsection{IR analysis.}

A different approach is a measurement of the quality of the overhead contact by using infrared cameras.

Main advantages offered by thermo vision are:

a) it is a non-contact and non-destructive technique;

b) it is suited to monitoring devices operating under high voltage or carrying high currents.

An IR imaging is more informative than a standard camera image, because of its insensitivity to different weather conditions, to the daily-nightly runs or to the presence of tunnels. Hot spots on the overhead line and on the pantograph strips are easy to detect and a careful analysis of the stored images can help maintenance operations in case of irregular positioning of the line (e.g., the wire should be zigzagged relative to the centre line of the track to even the wear on the train's pantograph as it runs underneath: in case of a defective layout of the line, the pantograph strip dramatically overheats and such effect is revealed from IR images). An interesting task is the setting-up of new methodologies for monitoring the wear of the pantograph strip. Wear primarily depends on the superficial temperature of the strip: this temperature, along the whole strip, can be qualitatively revealed, by processing the infrared image of the pantographcatenary interaction. Since pantograph is moving, the position of the strip changes in each frame of the video infrared sequence. Therefore, in order to detect automatically the temperature of the strip, a tracking algorithm must be employed. The problem of determining the motion of an object in image sequences is well known in literature. Because of the non-uniform elasticity of the contact wire, the pantograph has a motion along the vertical direction. Therefore, in order to estimate the dynamic profile of the temperature of the whole strip from a sequence of infrared images, a tracking algorithm of the strip has been implemented, based on the Radon-Hough transform (Deans, 2000). The shape of the strip is approximated 
as a segment: Hough transform is an effective technique for detecting line segments in a binary image (line-to-point transformation). The tracking algorithm is applied to the infrared image of the pantograph-catenary contact through the following steps:

- the image is pre-processed using edge detection algorithms, to produce a binary image,

- each non-zero image point is then transformed to a curve in the parameter space by using the Hough transformation (fig.8),

- the segment detected is highlighted on the original image (fig. 9.a),

- temperatures along the segment can be measured by a scanning procedure (fig. 9.b).

This procedure is repeated for each frame of the sequence. Since the pantograph moves slowly with respect to the frame rate of the camera, the position of the last strip detected is used for selecting a suitable window in the next image, in spite of reducing the computation time.

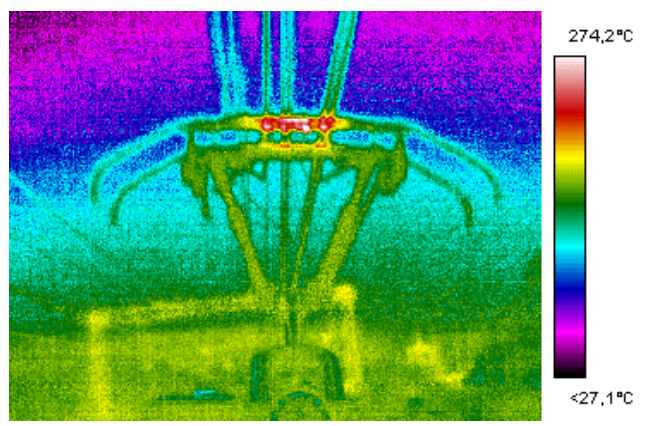

Fig. 7. IR image of a running pantograph.

Fig. 8 shows a visualization of the Hough transformation of the image. The transformation consists of several regions of high intensity values: coordinates of the highest peak are the parameters of the straight line representing the strip.

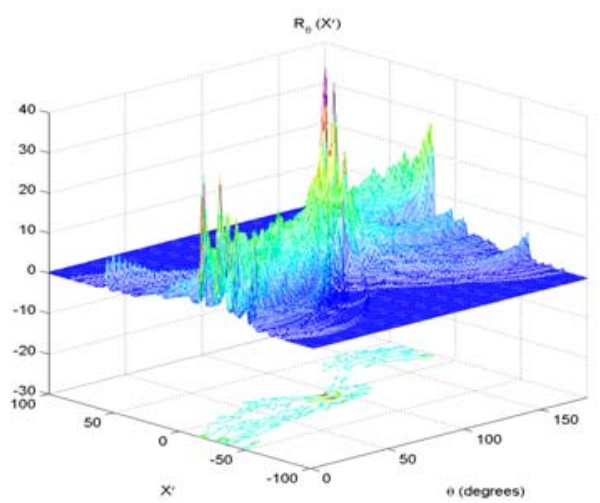

Fig. 8. Hough transformation of the image.

The dynamic temperature map of collector strips yields helpful information about the quality of the overhead contact. The obtained results are relevant for monitoring the status of the contact line and of the pantograph, for detecting critical points of the contact line and for planning maintenance activities.
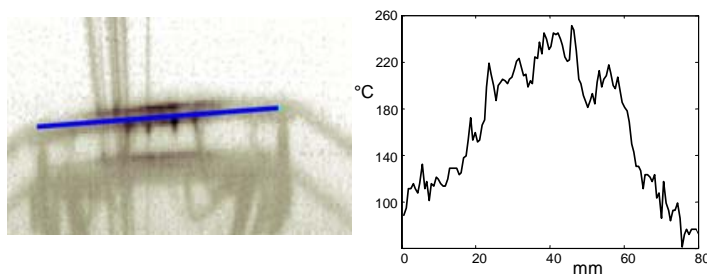

Fig. 9. a) Infrared image of the strip with detected line overlaid on the image b) Temperature plot along the detected segment.

\section{CONCLUSIONS}

Active control of pantographs for bettering the quality of current collection is a field of increasing interest in railway research. An effective monitoring of pantograph-catenary interactions is essential both for controlling the force exerted by the pantograph and for predictive maintenance. Solutions based on phototubes, wavelet analysis of tractive current, or IR camera are currently investigated and briefly described in the paper. Perspectives of the research activities are based on the study of minimal cost implementations of the solutions proposed in cooperation with railway companies.

\section{REFERENCES}

Balestrino, A., O. Bruno, A. Landi and L. Sani (2000). Innovative solutions for overhead catenary-pantograph system: wire actuated control and observed contact force. Vehicle System Dynamics, 33, 69-89.

Balestrino, A. et al..(2003). Infrared cameras for monitoring pantograph-catenary interactions. Proc. of the World Congress on Railway Research, CD-ROM, 1024-1029.

Barmada, S., A. Landi, M.Papi and L. Sani (2003). Wavelet multi-resolution analysis for monitoring the occurrence of arcing on overhead electrified railways. Proc. of the IMECHE, Part F: Journal of Rail and Rapid Transit, 217, 177-187.

Bruno, O., A. Landi, M.Papi and L. Sani (2001). Phototube sensor for monitoring the quality of current collection on overhead electrified railways. Proc. of the IMECHE, Part F: Journal of Rail and Rapid Transit, 215, 231-241.

Council Directive 96/48/EC of 23 July 1996 on the interoperability of the trans-European high-speed rail system. Official Journal L 235, 17/09/1996, 6-24.

Deans, S.R. (2000). Radon and Abel Transforms. In: The Transforms and applications Handbook (A.Poularikas (Ed.)). CRC Press.

Diana G., F. Fossati and F.Resta (1998). High speed railway: collecting pantographs active control and overhead lines diagnostic solutions. Vehicle System Dynamics, 30, 69-84.

Poetsch G. et al. (1997). Pantograph/catenary dynamics and control. Vehicle System Dynamics, 28, 159-195. 